\title{
Role of caffeine in DNA recognition of a potential food-carcinogen benzo[a]pyrene and UVA induced DNA damage
}

\author{
Soma Banerjee ${ }^{a}$, Siddhi Chaudhuri ${ }^{a}$, Anup Kumar Maity ${ }^{b}$, Partha Sahab \\ and Samir Kumar $\mathrm{Pal}^{\mathrm{a} *}$
}

\begin{abstract}
Electron transfer (ET) reactions are important for their implications in both oxidative and reductive DNA damages. The current contribution investigates the efficacy of caffeine, a xanthine alkaloid in preventing UVA radiation induced ET from a carcinogen, benzo[a]pyrene (BP) to DNA by forming stable caffeine-BP complexes. While steady-state emission and absorption results emphasize the role of caffeine in hosting BP in aqueous medium, the molecular modeling studies propose the energetically favorable structure of caffeine-BP complex. The picosecond-resolved emission spectroscopic studies precisely explore the caffeine-mediated inhibition of ET from BP to DNA under UVA radiation. The potential therapeutic activity of caffeine in preventing DNA damage has been ensured by agarose gel electrophoresis. Furthermore, time-gated fluorescence microscopy has been used to monitor caffeine-mediated exclusion of BP from various cell lines including squamous epithelial cells, WI-38 (fibroblast), MCF-7 (breast cancer) and HeLa (cervical cancer) cells. Our in vitro and ex vivo experimental results provide imperative evidences about the role of caffeine in modified biomolecular recognition of a model carcinogen BP by DNA resulting dissociation of the carcinogen from various cell lines, implicating its potential medicinal applications in the prevention of other toxic organic molecule induced cellular damages. Copyright $\odot 2014$ John Wiley \& Sons, Ltd.
\end{abstract}

Keywords: benzo[a]pyrene (BP); caffeine; inhibition of electron transfer (ET) reaction; prevention of DNA fragmentation; coexposure to UVA radiation and BP; picosecond-resolved fluorescence of BP; fluorescence microscopy; squamous epithelial cells; fibroblast (WI-38) cells; breast cancer (MCF-7) cells and HeLa cells

\section{INTRODUCTION}

Electron transfer (ET) reactions are notable for their significance in chemistry, biophysics, radiation biology and cancer biochemistry (Marcus, 1993; Lu, 2010; Fujitsuka and Majima, 2012; Banerjee et al., 2013a). A new concept of reductive DNA damage induced by ultrafast ET reactions of prehydrated electrons, a novel species of electrons produced by radiolysis of water under ionizing radiation, has emerged in some of the recent works (Wang et al., 2009; Nguyen et al., 2011). Such studies report that the effectiveness of prehydrated electrons induced reductive DNA damage is about twofold compared to that of oxidative DNA damage by hydroxyl radical. Some radiations lead to cellular damage by both direct and indirect interactions with the cell components. For example, UVB $(280-320 \mathrm{~nm})$ radiation is directly absorbed by DNA, which results in the formation of cyclobutane pyrimidine dimers (CPDs) and (6-4) photoproducts (Nielsen, 1990) and are responsible for the tumorigenic effects (de Gruijl, 2000). UVA ( $320-400 \mathrm{~nm}$ ) radiation, which is present more abundantly in sunlight compared to UVB, can induce various forms of DNA damage in the presence of endogenous or exogenous photosensitizers. It has recently been demonstrated that UVA can induce CPDs by direct absorption of DNA (Girard et al., 2011; Sage et al., 2012). Some of the earlier studies have reported that co-exposure of UVA radiation and benzo[a] pyrene (BP) can induce DNA damages like single-strand breaks and double-strand breaks (DSBs) in both in vivo and in vitro experimental conditions (Toyooka et al., 2004; Toyooka et al., 2006).
$\mathrm{BP}$ is a potential carcinogenic polyaromatic hydrocarbon (PAH) (Brown et al., 1996) to which people are frequently exposed through fuel and tobacco combustion along with grilled and smoked food products (Phillips, 1983; Banerjee et al., 2013a). Although BP is not carcinogenic per se and needs to be activated into a diolepoxide derivative, which covalently binds to DNA (at N2 and N7 of guanine and N3 of adenine), the possibility of direct intercalation of unmodified BP to DNA cannot be ruled out (Beck and Cramb, 2000). As one of our recent works highlights UVA radiation induced ultrafast ET from BP to DNA (Banerjee et al., 2013a), the DNA damages resulting from the co-exposure to BP and UVA radiation can be partially attributed to reductive DNA damage although other carcinogenic pathways involving reactive oxygen species causing oxidative DNA damages can also be involved. In our previous study (Banerjee

\footnotetext{
* Correspondence to: Samir Kumar Pal, Department of Chemical, Biological \& Macromolecular Sciences, S. N. Bose National Centre for Basic Sciences, Block JD, Sector III, Salt Lake, Kolkata 700 098, India.

E-mail: skpal@bose.res.in

a S. Banerjee, S. Chaudhuri, S. K. Pal

Department of Chemical, Biological \& Macromolecular Sciences, S. N. Bose National Centre for Basic Sciences, Block JD, Sector III, Salt Lake, Kolkata, 700 098, India

b A. K. Maity, P. Saha

Crystallography and Molecular Biology Division, Saha Institute of Nuclear Physics, Sector I, Block AF, Bidhannagar, Kolkata, 700064, India
} 
et al., 2013a), it has been proposed that ET pathway from BP to DNA can be targeted to prevent BP mediated DNA damage/ carcinogenesis, which is one of the main motives of this present work.

In order to target the UVA radiation induced ET pathway from $\mathrm{BP}$ to DNA, one of the most widely consumed xanthine alkaloid (Fredholm et al., 1999; Marta et al., 2010), 1,3,7-trimethylxanthine commonly known as caffeine, has been considered in the present study. Caffeine is known to inactivate essentially two proteins (ataxia-telangiectasia-mutated (ATM) and ATM-and Rad3-related), primarily responsible for the genome stability (Cortez, 2003). A number of earlier studies show that the alkaloid has some significant effect on the DNA recognition of intercalating drugs through "interceptor" and "protector" properties of caffeine (Traganos et al., 1991; Larsen et al., 1996 ; Davies et al., 2001). The specific molecular interaction of caffeine with different DNA-intercalating agents like doxorubicin, ethidium $(E t)$, acridine orange $(\mathrm{AO})$ and novantrone underlying in the "interceptor" action of caffeine results in the inhibition of cytostatic/cytotoxic effects of such intercalating drugs (Traganos et al., 1991 ; Larsen et al., 1996; Lyles et al., 2001). The "protector" activity of caffeine arises because of the competition between caffeine and other drug molecules for the same binding sites on DNA (Traganos et al., 1991; Davies et al., 2001). Some of our previous works emphasize the role of caffeine as an interceptor molecule, which helps to dissociate Et from biomimetic systems (Banerjee et al., 2012b), synthetic DNA and various cell nuclei (Banerjee et al., 2011). In another recent study, we have reported that caffeine can host some hydrophobic molecules like 4-(dicyanomethylene)2-methyl-6-(p-dimethylaminostyryl) 4H-pyran, coumarin 500 (C500) and 2-(p-toluidino) naphthalene-6-sulfonate by encapsulating them into its dimeric structure (Banerjee et al., 2012a). Such encapsulating property of caffeine due to its dimeric structure plays a key role in the solubilization of extremely hydrophobic BP molecules in the aqueous solvent (Boyland and Green, 1962; Nosaka et al., 1978). However, whether such solubilizing activity of caffeine can release BP from the DNA bound state, eventually preventing the ET from $B P$ to DNA under UVA radiation and the effect of caffeine on such $B P$ and UVA radiation induced reductive DNA damage are some of the areas that have never been explored and are the motives of this present work.

In this study, we have used steady-state emission and UV absorption spectroscopy to monitor the interaction of caffeine with BP molecules and molecular modeling studies to explore the energy-optimized geometry of the caffeine-BP complex. Steady-state and picosecond-resolved fluorescence spectroscopy have been used to monitor the effect of caffeine on ET reaction from $B P$ to DNA under UVA radiation, using a laser source of $375 \mathrm{~nm}$. Agarose gel electrophoresis (AGE) has been performed to monitor the DNA damage in terms of DNA DSBs by BP under UVA radiation in absence and presence of caffeine. Calf thymus (CT) DNA has been used for spectroscopic studies, whereas polymerase chain reaction (PCR) amplified DNA fragments of $\sim 1300 \mathrm{bp}$ have been used to monitor DNA damage through AGE. Time-gated fluorescence microscopy has been used to investigate the caffeine-mediated release of BP from various cell lines including squamous epithelial cells collected from the inner lining of the human mouth, WI-38 (fibroblast), MCF-7 (breast cancer) and HeLa (cervical cancer) cells in ex vivo conditions.

\section{MATERIALS AND METHODS}

\section{Sample preparation}

All chemicals used in this study were of analytical grade and were used without any further purification. Stock solution of benzo[a]pyrene (BP, obtained from Sigma) was prepared in dimethylsulfoxide (DMSO, from Spectrochem, Mumbai, India). CT-DNA (obtained from Sigma, St. Louis, Missouri, USA) solution was prepared in $50 \mathrm{mM}$ phosphate buffer ( $\mathrm{pH} 7.4$ ) while caffeine (Sigma) solution was prepared in double distilled water and phosphate buffer for spectroscopic and cellular studies, respectively. For steady-state fluorescence and UV-visible spectroscopic studies of BP in aqueous caffeine solutions, saturated solutions of BP were prepared by adding solid BP to caffeine solutions and kept under continuous stirring condition for $3 \mathrm{~h}$, and then the solutions were filtered to remove the excess BP, which did not dissolve. In the spectroscopic studies, DNA concentration is expressed in terms of base pair concentration. Nucleotide concentrations were determined by absorption spectroscopy using the average extinction coefficient per nucleotide of the DNA as $6600 \mathrm{M}^{-1} \mathrm{~cm}^{-1}$ at $260 \mathrm{~nm}$ (Pal et al., 2003). BP-DNA complexes were prepared as per the methodology reported previously (Geacintov et al., 1976) by Geacintov et al. In the fluorescence spectroscopic experiments of BP and CT-DNA, concentration of BP was $1 \mu \mathrm{M}$, while that of CT-DNA was $1 \mathrm{mM}$.

\section{Molecular modeling studies}

For the molecular modeling studies, we have followed the procedure as reported earlier for the calculation of caffeine hetero-dimer with a number of DNA-intercalating dyes (Larsen et al., 1996) and different hydrophobic dye molecules (Banerjee et al., 2012a). We have used commercially available ChemBio3D (from CambridgeSoft ${ }^{\mathrm{TM}}$, Cambridge, Massachusetts, USA) ultra software. The force field employed for these calculations is MM2 with default parameters provided with the software. Conjugate gradient methods were used to search for geometry-optimized structures with a convergence criterion of $0.0001 \mathrm{kcal} / \AA \AA$. The minimized energy values of the various complexes as investigated in the present study (caffeinecaffeine, and caffeine-BP) were obtained by first geometry optimizing each component of the complex in vacuum. These geometry-optimized components were then brought together in a face to face orientation and to within van der Waals radii and re-optimized. Relative binding energies were determined by subtracting the sum of the geometry-optimized energies of the isolated components from the total energy obtained for the geometry-optimized complex. It must be pointed out that the orientation of the caffeine relative to the dyes in the geometry-optimized complexes is not unique. For example, small displacement $(\sim 1 \AA)$ and rotation of the caffeine by $180^{\circ}$ relative to a dye ring system does not significantly alter the relative binding energy. The relative binding energies derived from molecular mechanics methods can be further deconvoluted into individual components that make up the total energy. In our present study, we like to be limited in the overall stabilization energy of the complexes as shown in Table 1. However, we have recognized that the dominant force in the formation of such complexes appears to be van der Waals interactions resulting in maximal ring overlap between the two molecules of the complexes. 
Table 1. Parameters from molecular modeling of caffeine-BP complex

\begin{tabular}{|c|c|c|c|}
\hline Complex & $\begin{array}{l}\text { Total energy }{ }^{\mathrm{a}} \\
\text { (E) (kcal/mole) }\end{array}$ & $\begin{array}{l}\text { Complex energy } \\
\quad(\mathrm{kcal} / \mathrm{mole})\end{array}$ & $\begin{array}{c}\Delta \mathrm{E}^{\mathrm{c}} \\
\text { (kcal/mole) }\end{array}$ \\
\hline Caffeine-caffeine & 52.62 & 42.34 & -10.28 \\
\hline Caffeine-BP & 0.92 & -10.5 & -11.42 \\
\hline Caffeine-BP-caffeine & 27.23 & 3.73 & -23.5 \\
\hline
\end{tabular}

\section{Steady-state and time-resolved measurements}

Steady-state absorption and emission were measured with Shimadzu Model UV-2450 spectrophotometer (Shimadzu Corporation, Tokyo, Japan) and Jobin Yvon Model Fluoromax-3 fluorimeter (Horiba Scientific, Stanmore, UK), respectively. All picosecond transients were measured by using commercially available (Edinburgh Instrument, UK) picosecond-resolved time correlated single-photon-counting setup (instrument response function, IRF of $80 \mathrm{ps}$ ) using $375 \mathrm{~nm}$ excitation laser source. Fluorescence from the sample was detected by a photomultiplier after dispersing through a double grating monochromator. For all transients, the polarizer in the emission side was adjusted to be at $54.7^{\circ}$ (magic angle) with respect to the polarization axis of the excitation beam.

Curve fitting of observed fluorescence transients was carried out using a nonlinear least-square fitting procedure to a function $\left(X(t)=\int_{0}^{t} E\left(t^{\prime}\right) R\left(t-t^{\prime}\right) d t^{\prime}\right)$ composed of convolution of the IRF $(\mathrm{E}(\mathrm{t}))$ with a sum of exponentials $\left(R(t)=A+\sum_{i=1}^{N} B_{i} e^{-t / \tau_{i}}\right)$ with pre-exponential factors $\left(B_{i}\right)$, characteristic lifetimes $\left(\tau_{\mathrm{i}}\right)$ and a background $(A)$. Relative concentration in a multi-exponential decay is expressed as

$$
c_{n}=\frac{B_{n}}{\sum_{i=1}^{N} B_{i}} \times 100
$$

The average lifetime (amplitude-weighted) of a multiexponential decay (Lakowicz, 1999) is expressed as

$$
\tau_{\mathrm{av}}=\sum_{i=1}^{N} c_{i} \tau_{i}
$$

After the deconvolution, obtained time constants, which are one-fourth of the IRF, may be reliably reported within the signal-to-noise ratio of the fluorescence transients.

\section{Preparation of DNA fragment}

For the preparation of DNA fragments, a 1300-bp sequence of Origin Recognition Complex (ORC1) DNA from Leishmania donovani was amplified from pGAT2ORC1 plasmid by PCR (Eppondorf, Germany) using primers LdORC1R1+ (5'GCAC TGA ATT CAA ATG AAG CGG AGC CGG CGA GC 3') and LdORC1Not1.1(5' GCT TCT GCG GCC GCC AAG TGC AGC GCT GCC CCG T 3'). The $\mathrm{PCR}$ product was purified using PCR clean up kit (Axygen, USA).

\section{Exposure to BP and/or UVA radiation}

DNA fragments (200 ng) dissolved in phosphate buffer, BP $\left(\sim 3 \times 10^{-4} \mathrm{M}\right)$ from stock DMSO solution and caffeine $\left(4 \times 10^{-2} \mathrm{M}\right)$ from stock solution were added and mixed thoroughly. Similarly, three other solutions were prepared containing only DNA, DNA with caffeine and DNA with BP. In the solutions containing only DNA and DNA with caffeine, equivalent amount of DMSO was added without BP. The four different sets of solutions were placed in a 96-well plate and irradiated with several doses of UVA $\left(\sim 400 \mathrm{~J} / \mathrm{cm}^{2}\right)$ using a UV source (Professional 54 W Shellac Gelish Curing machine from SiSi Business, USA) with an exposure time of $45 \mathrm{~min}$. The emission characteristic of the UV source has been checked with fiber optic cable connected to a spectrometer (from Ocean Optics, Dunedin, Florida, USA), which shows a major contribution at $365 \mathrm{~nm}$ along with some negligible or minimal contributions at 313 and $334 \mathrm{~nm}$ (see below). As a control experiment, similar sets of solutions were prepared where UVA doses were not delivered.

\section{Detection of double-strand breaks in the DNA fragment}

DNA fragments treated without and with BP and/or UVA in absence and presence of caffeine were loaded in a $0.8 \%$ agarose gel with loading buffer $(0.25 \%$ each of bromophenol blue and xylene cyanol $\mathrm{FF}$ and $30 \%$ glycerol in water, $\mathrm{pH}$ 7.6) and electrophoresed. The composition of running buffer was $0.045 \mathrm{M}$ Tris, $0.045 \mathrm{M}$ Boric acid and $1 \mathrm{mM}$ EDTA, pH 8.0. The gel was stained with ethidium bromide and visualized using a UV-transilluminator (Spectroline, USA) with $312 \mathrm{~nm}$ light source.

\section{Cells and cell culture conditions}

Squamous epithelial cells were collected from the inner lining of the human mouth. WI-38 (fibroblast), MCF-7 (breast cancer) and HeLa cells were grown in Dulbecco's modified Eagle's medium supplemented with $10 \%$ fetal bovine serum and penicillinstreptomycin $(0.5 \mathrm{U} / \mathrm{ml}$ of penicillin and $0.5 \mu \mathrm{g} / \mathrm{ml}$ streptomycin) in $35 \mathrm{~mm}$ dishes at $37^{\circ} \mathrm{C}$ in an atmosphere of air with $5 \% \mathrm{CO}_{2}$ and constant humidity.

\section{Fluorescence microscopy studies}

Fluorescence micrographs of squamous epithelial cells were taken using an Olympus BX51 fluorescence microscope connected with DP72 microscope digital camera, while those of other cell lines were taken using Zeiss AxioObserver Fluorescence Microscope (Oberkochen, Germany) attached with an Apotome apparatus. The Olympus fluorescence microscope is equipped with various sets of fluorescence mirror unit combined with appropriate filters that are variable depending on wavelengths. The fluorescence mirror unit that matches the fluorochrome in use (BP) was selected. All the cells were stained with $20 \mu \mathrm{M}$ BP for $15 \mathrm{~min}$, and excess BP was washed out with 
phosphate buffer. Cells were irradiated under UV light at $360 \mathrm{~nm}$ $(18 \mathrm{~mW})$ from a mercury bulb attached with the fluorescence microscope, and micrographs were taken at $10 \mathrm{~s}$ interval for 3-4 min with $(+)$ and without $(-)$ the addition of caffeine $(\sim 10 \mathrm{mM})$ and the time scale within which maximum change has been observed is reported for different cell lines. Squamous cell images were taken under 50x magnification, while those of other cell lines were taken using 40x magnification. The micrographs were analyzed with analySIS Five (Olympus, Nottinghill, Australia) image analysis software provided with the microscope. The software was used (RGB analysis mode) to measure the intensity of blue component in each micrograph.

Time-resolved relative emission intensity of BP inside the cells in control set of studies (- caffeine) has been determined by monitoring the emission intensity of BP inside the cells at different times $(t)$ with respect to the same at initial time $\left(t_{0} ; 10 \mathrm{~s}\right)$ just after the irradiation of the cells. The relative emission intensity of BP inside the cells after the addition of caffeine (+ caffeine) has been monitored by measuring the emission intensity of BP inside the cell with respect to that outside the cell at different times upon the caffeine treatment.

\section{RESULTS AND DISCUSSIONS}

\section{Caffeine-BP complexation in aqueous medium: structural characterization}

Figure 1(a) shows the molecular structures of caffeine and benzo[a] pyrene (BP) whose associations are reflected in Figure 1(b and c). Figure 1(b) shows the relative fluorescence intensity of BP dissolved maximally in aqueous caffeine solutions with varied caffeine concentration (ranging from 0 to $100 \mathrm{mM}$ ). Presence of three well characterized emission peaks of BP at 410, 430 and $455 \mathrm{~nm}$ as shown in Figure 1(b) with no signature of broad emission feature that peaks around $490 \mathrm{~nm}$ characteristic for BP aggregates (Beck and Cramb, 2000; Banerjee et al., 2013a) suggests that BP in caffeine solutions are present primarily as monomers. As evident from Figure 1(b), the fluorescence intensity of BP increases with enhancement of caffeine content in the aqueous solutions, which is in close agreement with the previously reported studies (Boyland and Green, 1962) emphasizing the role of caffeine in solubilizing BP. The caffeine-mediated solubilization of BP in aqueous medium has been further studied by UV-visible absorption spectra of the solutions upon diluting them 11 times with caffeine solutions of equivalent concentrations as shown in Figure 1(b) upper inset. The purpose of diluting the BP content in the caffeine solution is to keep the optical density (O.D.) values of BP much below 1 to avoid significant light scattering for the proper calculation of BP concentration from its molar extinction coefficient as per the Lambert Beer law (Villringer and Chance, 1997). The multiple peaks in the absorption spectra of BP in caffeine solutions at 330, 349, 368 and $388 \mathrm{~nm}$, as shown in Figure 1(b) upper inset, are assigned to the individual electronic transitions of BP as reported earlier (Larsen et al., 1992). The enhancement in the O.D. values of BP with the increase in caffeine concentration clearly indicates that the number of BP molecules in aqueous medium is directly proportional to the number of caffeine molecules in it. Figure 1(b) lower inset shows the solubility of BP in aqueous caffeine solution as a function of caffeine concentration, monitored from the absorption spectra of BP using molar extinction coefficient that has been calculated from its known concentration in similar

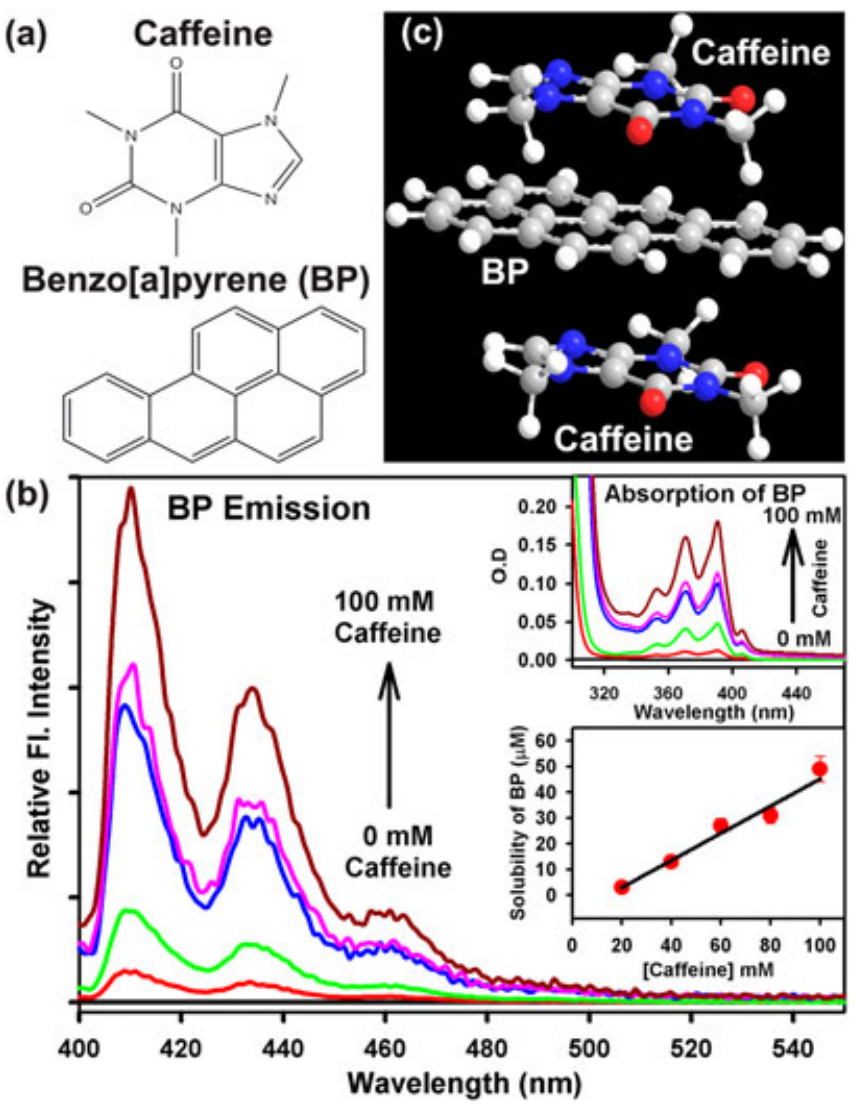

Figure 1. (a) Molecular structure of caffeine and benzo[a]pyrene (BP). (b) The fluorescence spectra of BP solubilized in aqueous caffeine solution with caffeine concentration varying from 0 to $100 \mathrm{mM}$; the upper inset shows the UV-visible absorption spectra of the same, while the lower inset shows the solubility of BP at different caffeine concentrations as obtained from absorption measurements. (c) The energy minimized structure of caffeine-BP complex obtained from the molecular modeling studies (see text).

medium. While calculating the concentration of BP in the caffeine solutions, the dilution factor has been considered. The plot of BP solubility versus caffeine concentration, calculated from the O.D. values of BP as shown in Figure 1(b) lower inset, holds well to the one reported previously from the fluorescence intensities of the same(Boyland and Green, 1962). Caffeine-mediated solubilization of extremely hydrophobic BP molecules in aqueous medium can be explained in terms of complexation between the two as previously observed for 4-(dicyanomethylene)-2-methyl-6-( $p$ dimethylaminostyryl) 4H-pyran molecules (Banerjee et al., 2012a), where it has been reported that caffeine can host some hydrophobic molecules in aqueous medium because of its dimeric nature. In this regard, we performed some molecular modeling calculations to find a mechanistic model of confinement of BP molecules within the caffeine dimer as shown in Figure 1(c). As shown in Table 1, our molecular modeling studies reveal a stable stacked dimer of caffeine molecules with stabilization energy of $\sim 10 \mathrm{kcal} / \mathrm{mole}$, which is consistent with previous reports (Carlucci and Gavezzotti, 2005; Banerjee et al., 2012a). The molecular modeling studies suggest that at lower caffeine concentration, 1:1 caffeine-BP hetero-dimer is stable with stabilization energy comparable to that of caffeine dimer, while at higher caffeine concentration, a ternary complex of two caffeine molecules with one BP molecule is much more stable with 
higher stabilization energy as shown in Table 1 and Figure 1(c). The structure of caffeine-BP complex as derived from our molecular modeling studies (Figure $1(\mathrm{c})$ ) is consistent with the stacking sandwich type model (caffeine-BP-caffeine) proposed previously from NMR experiments (Nosaka et al., 1978).

\section{Effect of caffeine on electron transfer reaction from BP to DNA under UVA radiation}

Upon monitoring the interaction between caffeine and BP in aqueous medium, the effect of caffeine on DNA bound BP has
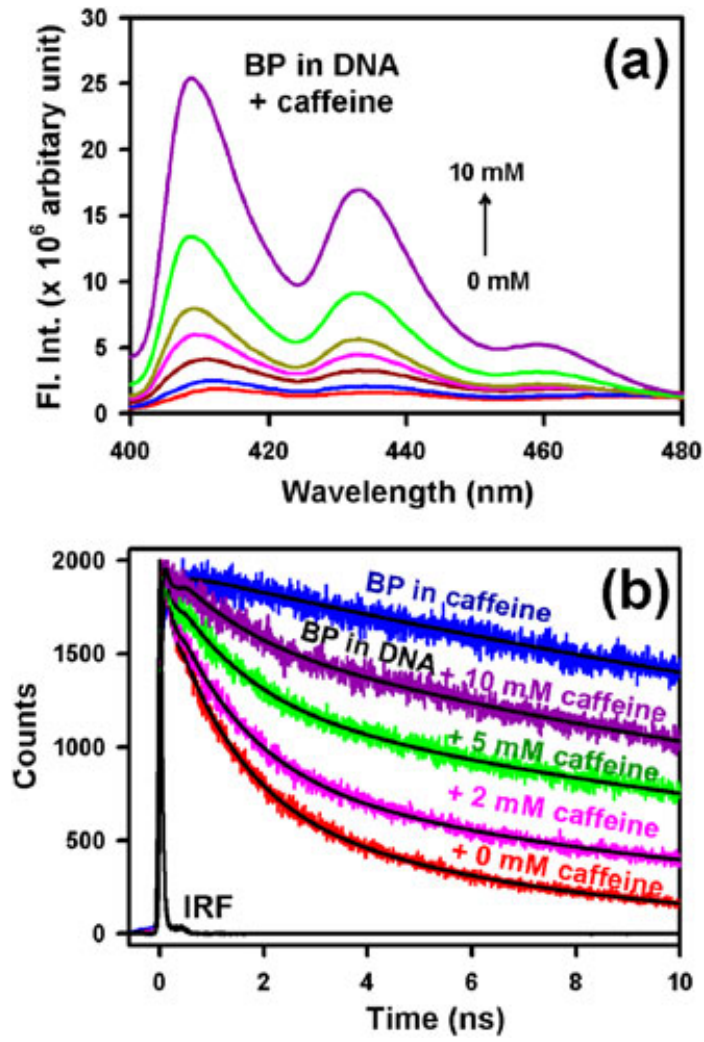

Figure 2. (a) The enhancement in the fluorescence intensity and (b) lifetime of BP in CT-DNA with the increase in caffeine concentration from 0 to $10 \mathrm{mM}$ along with the fluorescence transient of BP in caffeine solution. Fluorescence transients have been monitored $\left(\lambda_{\mathrm{em}}\right)$ at $410 \mathrm{~nm}$. Excitation wavelength $\left(\lambda_{\mathrm{ex}}=375 \mathrm{~nm}\right)$. been monitored through steady-state and picosecond-resolved fluorescence spectroscopy. Figure 2(a) shows that the emission intensity of DNA bound BP upon exciting with UVA radiation enhances with the increase in caffeine concentration from 0 to $10 \mathrm{mM}$. As our previous study (Banerjee et al., 2013a) suggests that $\mathrm{ET}$ reaction takes place from $\mathrm{BP}$ to DNA under UVA radiation, the enhancement in emission intensity of the DNA bound BP in presence of caffeine indicates the possibility of caffeinemediated inhibition of such nonradiative ET pathway. For further confirmation of the role of caffeine in inhibiting the mentioned ET reaction, fluorescence lifetimes of BP in presence of DNA under UVA radiation, at different caffeine concentrations, have been monitored at $410 \mathrm{~nm}$ as shown in Figure 2(b), and the decay parameters are presented in Table 2. The purpose of monitoring the fluorescence transients at $410 \mathrm{~nm}$ lies under the fact that the contribution of $\mathrm{BP}$ aggregates is minimum or negligible at $410 \mathrm{~nm}$, and therefore, the fluorescence originates essentially from the BP monomers (Banerjee et al., 2013a). The fluorescence lifetime of DNA bound BP molecules under UVA radiation in absence of caffeine as shown in Figure 2 (b) and Table 2 is consistent with the one reported in our previous study (Banerjee et al., 2013a). Figure 2(b) and Table 2 show that the contribution of ET components (70 and $900 \mathrm{ps)}$ (Banerjee et al., 2013a) in the fluorescence lifetime of BP gradually decreases with the increase in caffeine concentration along with the manifestation of a longer lifetime component of $30 \mathrm{~ns}$, which is similar to the lifetime of BP upon complexation with caffeine in aqueous medium as shown in Figure 2(b) and tabulated in Table 2. The values of the mentioned time components have been fixed in the fitting of the fluorescence transients to monitor the variation in the relative contribution of the components with increase in caffeine concentration. Gradual increase of $30 \mathrm{~ns}$ component of DNA-BP system in presence of increasing concentration of caffeine clearly reveals detachment of BP from DNA to the caffeine moiety in aqueous solution. The observation is consistent with the fact that the ET processes of BP in the DNA moiety is severely blocked when BP is detached from DNA upon complexation with caffeine. By measuring the relative contribution of the longer lifetime component $(30 \mathrm{~ns})$, which is a signature of the total population of BP bound to caffeine upon releasing from DNA, and knowing the total concentration of DNA bound BP ([BP]) and caffeine ([Caffeine]) in the solution, we have calculated the relative association constant $(K)$ between caffeine and BP in aqueous DNA solution using the following equation (Sarkar and Pal, 2006),

Table 2. The lifetime components of BP in CT-DNA at different caffeine concentrations monitored at $410 \mathrm{~nm}\left(\lambda_{\mathrm{em}}\right)$

\begin{tabular}{|c|c|c|c|c|c|c|}
\hline Sample & Caffeine (mM) & $\tau_{1}(\mathrm{~ns})$ & $\tau_{2}(\mathrm{~ns})$ & $\tau_{3}(\mathrm{~ns})$ & $\tau_{4}(\mathrm{~ns})$ & $<\tau_{\text {avg }}>$ (ns) \\
\hline \multirow[t]{7}{*}{ BP in DNA solution } & 0 & 0.07 (32\%) & $0.90(37 \%)$ & $5.61(31 \%)$ & & 2.10 \\
\hline & 0.5 & $0.07(33 \%)$ & $0.90(31 \%)$ & $4.34(30 \%)$ & $30.00(6 \%)$ & 3.29 \\
\hline & 1 & $0.07(32 \%)$ & $0.90(30 \%)$ & 4.37 (30\%) & $30.00(8 \%)$ & 4.02 \\
\hline & 2 & $0.07(32 \%)$ & $0.90(24 \%)$ & $3.72(27 \%)$ & $30.00(17 \%)$ & 6.30 \\
\hline & 3 & $0.07(25 \%)$ & $0.90(32 \%)$ & $5.61(23 \%)$ & $30.00(20 \%)$ & 7.69 \\
\hline & 5 & $0.07(24 \%)$ & $0.90(22 \%)$ & $5.61(20 \%)$ & 30.00 (34\%) & 11.49 \\
\hline & 10 & $0.07(23 \%)$ & $0.90(12 \%)$ & $5.61(15 \%)$ & $30.00(50 \%)$ & 15.98 \\
\hline BP in caffeine solution & & - & - & - & $30.00(100 \%)$ & 30.00 \\
\hline
\end{tabular}




$$
K=\frac{[\text { Caffeine }-B P]}{([B P]-[\text { Caffeine }-B P]) \times([\text { Caffeine }] .-[\text { Caffeine }-B P])}
$$

where $[$ Caffeine-BP], ([BP] - [Caffeine-BP]), ([Caffeine] - [Caffeine-BP]) represent concentration of Caffeine-BP complex, BP bound to DNA and free Caffeine in the solution, respectively. The relative association constant of BP with caffeine is calculated to be $\sim 100 \mathrm{M}^{-1}$, which is comparable with the association constant between caffeine and a well known DNA-intercalating dye ethidium (Et) (Larsen et al., 1996; Banerjee et al., 2011). It has to be noted that molecular recognition of Et/BP depends on the length of the host DNA (Sarkar and Pal, 2006). DNA recognition of Et/BP upon DNA fragmentation requires further investigation and is the motive of our future work.

\section{Role of caffeine in the prevention of BP and UVA induced DNA damage}

The caffeine-mediated release of BP from DNA, consequently inhibiting the UVA radiation induced ET from BP to DNA as observed from our fluorescence spectroscopic studies, motivated us to find the efficacy of caffeine in the prevention of BP and UVA radiation induced DNA damage. In this regard, $1300 \mathrm{bp}$ DNA fragments were co-exposed to BP and UVA in absence ( - ) and presence ( + ) of caffeine, and the formation of DSBs in the DNA was monitored by AGE as shown in the upper panel of Figure 3. As evident from Figure 3, co-exposure of the DNA fragments to BP and UVA (lane G) results in the formation of a smear around $250 \mathrm{bp}$ as shown by the dotted arrow, and the result is in close agreement with earlier studies (Toyooka et al., 2006). Given the composition of the loading buffer (Materials and Methods section), it is unlikely that the buffer could denature the DNA under study. In addition, the intactness of the ds DNA marker under the same loading buffer also rules out the possibility of DNA denaturation. Thus, the fragments obtained in lane G can be considered as DSBs and not singlestrand breaks. However, in presence of caffeine (lane $H$ ), the linear DNA fragments produce their corresponding band (shown by bold arrow in Figure 3) at around 1300 bp in accordance with the DNA marker and no smaller fragments of DNA because DSBs are observed, which emphasizes the efficacy of caffeine in preventing the DSBs in DNA. One of the possible mechanisms imparting caffeine such preventive role against DNA damage can be the inhibition of ET from BP to DNA. Here, it has to be noted that the absence of sharp DNA bands in lanes $G$ and $H$ upon co-exposure to BP and UVA even in presence of caffeine is because intercalation of BP into the DNA prevents ethidium (Et) in the agarose gel to get into the DNA to produce the sharp bands of DNA bound Et under UV-transilluminator. Therefore, the weak bands are due to the DNA bound BP, which also fluoresces blue-green under the transilluminator. Such inference has been drawn upon repetition of the aforementioned experiment with visualization under transilluminator both before and after staining with Et, which produced blue-green emission of BP from the DNA bands in lanes $\mathrm{G}$ and $\mathrm{H}$. For better understanding of the obtained results, we have employed steady-state spectroscopy to monitor the binding affinity of Et towards DNA upon exposure to UVA radiation both in absence and presence of BP (data not shown) and found that the binding affinity of Et to DNA decreases upon co-exposure of BP and UVA. Such $\mathrm{BP}$ and UVA induced reduction in the binding of Et to DNA may be rationalized in terms of significant perturbation of DNA

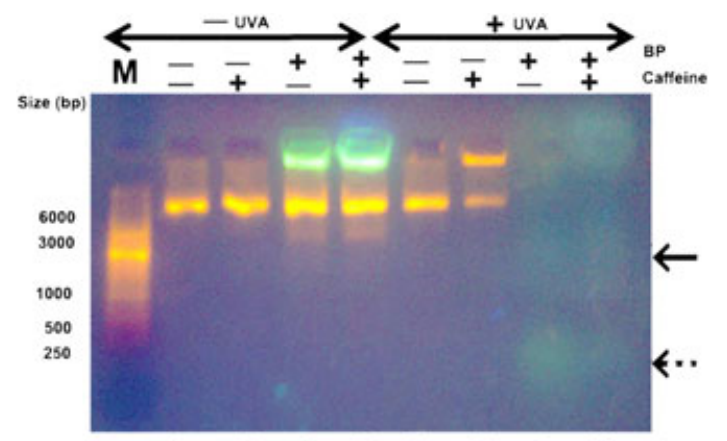

A B C D E F

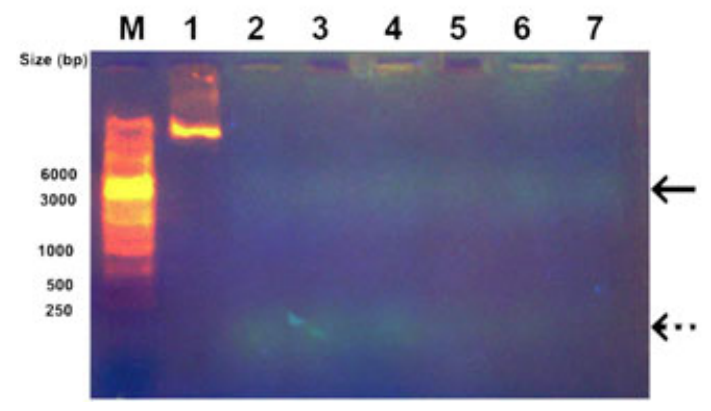

Figure 3. Upper Panel. DNA fragments of $1300 \mathrm{bp}$ were exposed to $\left(3 \times 10^{-4} \mathrm{M}\right)$ BP with UVA radiation $\left(\sim 400 \mathrm{~J} / \mathrm{cm}^{2}\right)$ (lanes $\mathrm{G}$ and $\mathrm{H}$ ) in absence (lane $\mathrm{G}$ ) and presence of caffeine (lane $\mathrm{H}$ ), loaded onto a $0.8 \%$ agarose gel and electrophoresed. DNA fragments without caffeine (lane E) and with caffeine (lane F) in presence of UVA radiation were also loaded and electrophoresed. Similar experiments without UVA radiation were commenced (lanes A, B, C and D) as control. The absence and presence are represented with the symbols $(-)$ and $(+)$, respectively for UVA radiation, $\mathrm{BP}$ and caffeine. $\mathrm{M}$ represents the lane loaded with DNA marker of varying sizes (in base pairs). The fragmented DNA resulting from double-strand breaks (DSBs) ( 200-250 bps) is shown in dotted arrow, while the bold arrow represents the 1300 bp DNA without any DSBs. The gel was stained with ethidium bromide and all the experiments had been repeated five times with similar results. Lower Panel. DNA fragments of $1300 \mathrm{bp}$ exposed to $\left(3 \times 10^{-4} \mathrm{M}\right)$ BP (lanes 2-7) with UVA radiation $\left(\sim 400 \mathrm{~J} / \mathrm{cm}^{2}\right)$ with varying caffeine concentration $0.0,0.1,0.5,1.0,5.0$ and $10.0 \mathrm{mM}$, respectively. Lane 1 contains similar DNA fragments exposed only to UVA radiation $\left(\sim 400 \mathrm{~J} / \mathrm{cm}^{2}\right)$ without BP and caffeine, as a control. M represents the lane loaded with DNA marker of varying sizes (in base pairs). The fragmented DNA resulting from double-strand breaks (DSBs) $(\sim 200-250$ bps $)$ is shown as dotted arrow. The gel was stained with ethidium bromide, and all the experiments had been repeated three times with similar results.

structure including the formation of CPDs, 8-oxoguanine and single/double-strand breaks, which invite further investigation. In presence of caffeine, BP does not get completely released from DNA, but the effective concentration of BP in the DNA decreases, which is enough to prevent the DSBs. As a control set of experiments, DNA fragments have been exposed only to BP without UVA dose (lane C), which result in no DSBs holding well to the fact that DSBs form under the co-exposure of BP and UVA (Toyooka et al., 2006). Sharp DNA bands produced by DNA intercalated Et in the gel upon exposing the DNA fragments to BP without UVA dose (lane C) signify the weaker association of BP with DNA, which is released from the DNA during electrophoresis. Excess BP and caffeine-BP complex which are not bound to DNA remain in the loading wells of the agarose gel (lanes $C, D, G$ and $H$ ) and do not migrate during electrophoresis as they carry no charge. As BP and Et 
have comparable spectral overlaps of their absorption coefficients with the transilluminator emission at $312 \mathrm{~nm}$, both the dyes have similar absorption of photons from the said source. On the other hand, the fluorescence quantum yield of BP is around unity (Banerjee et al., 2013b), while that of DNA bound Et (Le Pecq, 2006) is $\sim 0.15$, which is the reason behind the extensive fluorescence of BP under the transilluminator. However, BP undergoes through nonradiative ET pathway upon associating with DNA. As a result, the emission intensity of excess BP and BP-caffeine complexes remaining in the wells is much higher compared to that of BP molecules associated with the DNA bands. Our control experiments where DNA has been exposed only to caffeine in presence (lane F) and absence of UVA radiation (lane $B$ ) show that caffeine does not cause any significant change in the DNA, which can be reflected through the gel electrophoresis. Furthermore, our control experiments suggest that only UVA radiation has some effect on the DNA fragments because of which a portion of DNA is stuck into the loading well (lane E). Such retardation of DNA migration is known to arise from DNA-DNA cross-links (Lu and Morimoto, 2009). Lower panel of Figure 3 shows DNA fragments of $1300 \mathrm{bp}$ exposed to $\left(3 \times 10^{-4} \mathrm{M}\right) \mathrm{BP}$ (lanes 2-7) with UVA radiation $\left(400 \mathrm{~J} / \mathrm{cm}^{2}\right)$ with varying caffeine concentration $0.0,0.1,0.5,1.0,5.0$ and $10.0 \mathrm{mM}$, respectively. Lane 1 contains similar DNA fragments exposed only to UVA radiation $\left(\sim 400 \mathrm{~J} / \mathrm{cm}^{2}\right)$ without $\mathrm{BP}$ and caffeine, as a control. $M$ represents the lane loaded with DNA marker of varying sizes (in base pairs). The fragmented DNA resulting from DSBs ( 200-250 bps) is shown as dotted arrow. The intensity of DSBs fragment as indicated by the dotted arrow gradually decreases with the increase in caffeine concentration.

To study the mechanism of such cross-link formation, the absorption spectra of DNA and the plastic material of 96-well plate, in which the experiments were carried out, have been monitored as shown in Figure 4(a), and the emission intensity of the used UV source has also been checked within the same wavelength range as shown in Figure 4(b). As evident from Figure 4(b), along with the major emission peak at $365 \mathrm{~nm}$, there are negligible or 100 times less intense contribution of the UV source at 313 and $334 \mathrm{~nm}$. Some earlier works (Girard et al., 2011) suggest that low energy of UVA photons $(322-390 \mathrm{~nm})$ can be directly absorbed by DNA and are sufficient to produce CPDs usually in the form of thymine-thymine cross-links, yet at a low level. The observed DNA-DNA crosslinks can also occur indirectly through the formation of hydrogen peroxide $\left(\mathrm{H}_{2} \mathrm{O}_{2}\right)$. One of the previous studies (Kuluncsics et al., 1999) found that UVA irradiation of DNA solution or phosphate buffer alone on plastic Petri dishes can result in the formation of $\mathrm{H}_{2} \mathrm{O}_{2}$. The formation of $\mathrm{H}_{2} \mathrm{O}_{2}$ on plastic plates can be explained on the basis of the absorption spectrum of the plastic as shown in Figure 4(a) (blue line) over the wavelength range of UVA radiation $(320-399 \mathrm{~nm})$. As plastic has significant absorbance in the UVA region, it can act as a sensitizer and expected to produce reactive oxygen species like $\mathrm{H}_{2} \mathrm{O}_{2}$ that may result in oxidative damage of DNA (Cadet et al., 2009) and DNA-DNA cross-links (Colis et al., 2008). Interestingly, such phenomenon of DNA-DNA cross-links is not observed in the agarose gel when the DNA fragments are exposed to UVA radiation in presence of caffeine (lane $\mathrm{F}$ of Figure 3). The relatively high concentration of caffeine molecules in the experimental system compared to DNA results in absorption of UVA radiation by caffeine to a greater extent (as shown in Figure 4(a), pink line) and acts

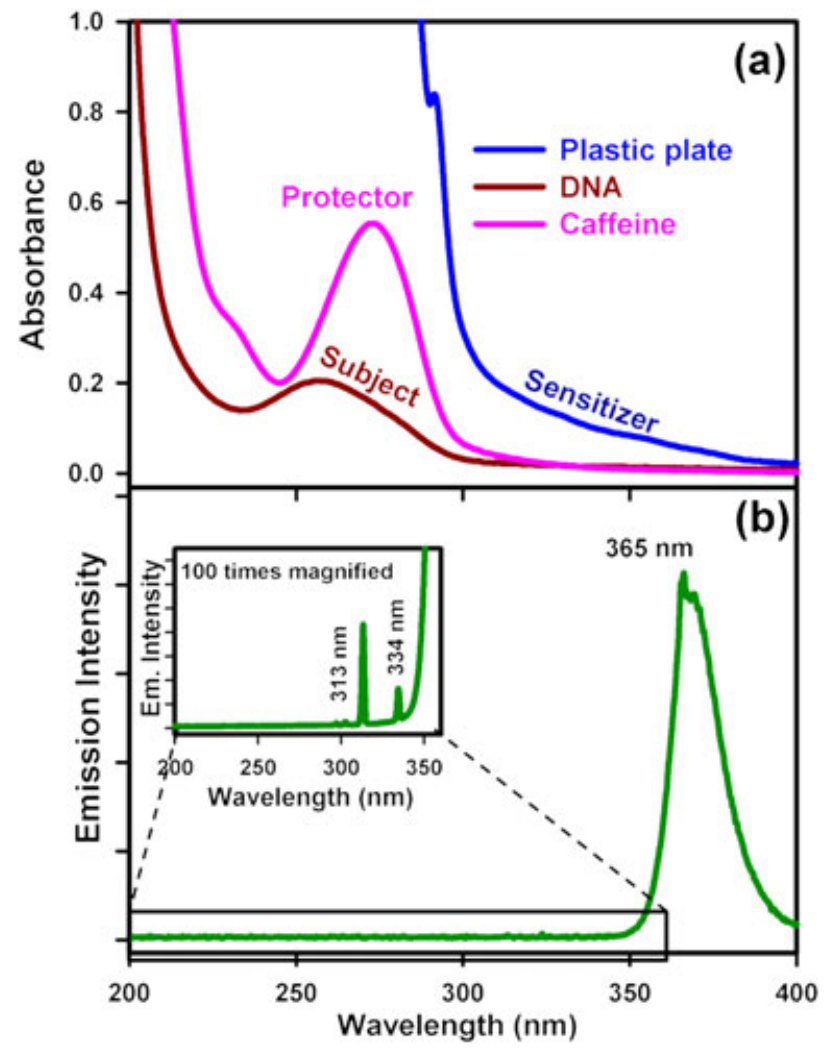

Figure 4. (a) UV absorption spectra of DNA (subject), caffeine (protector) and plastic material of the 96-well plate (sensitizer). See text for details. (b) Emission intensity of the UV source within the same wavelength range, and inset shows the 100 times magnified image of a portion of the spectra marked by a rectangle.

as a protector preventing the formation of DNA-DNA crosslinks by direct absorption of UVA photons by DNA. Another possible mechanism through which caffeine can prevent DNA-DNA cross-linking is through its free radical scavenging activity (Rafael et al., 2011). However, the significant amount of DNA, which is stuck to the loading well in the agarose gel as observed from lane $\mathrm{E}$ of upper panel of Figure 3, may not be solely due to the formation of intermolecular cross-links. In this regard, the possibility of the DNA being contaminated with the products extracted from the purification column can be ruled out, as we did not observe such attachment of DNA to the wells of other lanes in the agarose gel. The different types of DNA damages associated with such exposure of DNA to the UVA source can be preferably found out using a DNA plasmid assay employing DNA repair enzymes, which is one of the motivations of our future works.

\section{Caffeine-mediated exclusion of BP from various cell lines: cellular studies under ex vivo condition}

The in vitro experimental results emphasizing the role of caffeine in releasing BP from DNA upon forming caffeine-BP complex have been further verified in biological milieu using different animal cell lines. Figure 5(a) shows the fluorescence micrographs of BP-stained squamous epithelial cells (collected from the inner 

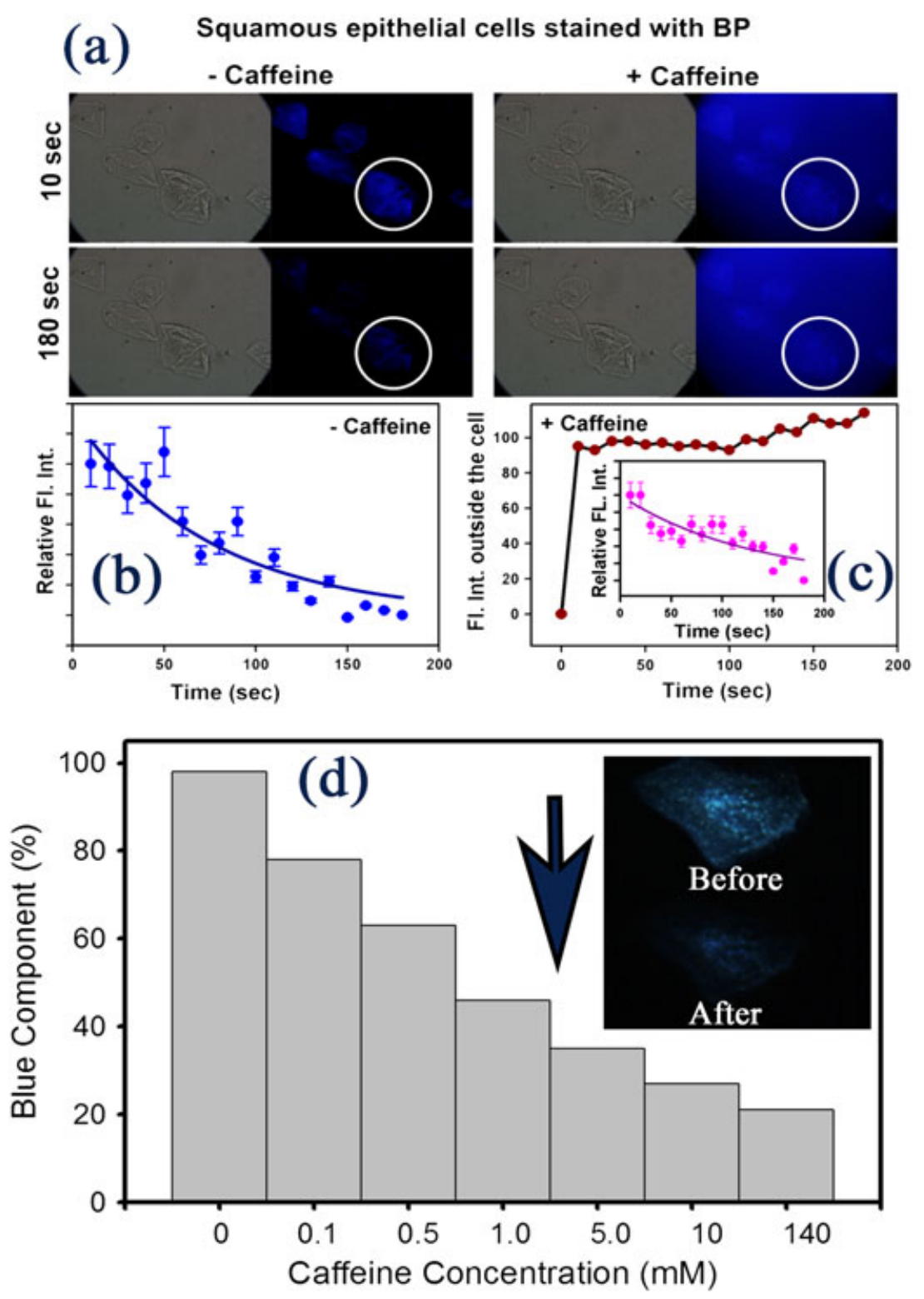

Figure 5. (a) Fluorescence micrographs of BP-stained squamous epithelial cells taken at 10 and $180 \mathrm{~s}$ after the addition of caffeine (+ caffeine). Images in grayscale show the respective bright field micrographs. As a control set of experiments, micrographs were taken at similar timescales upon the addition of buffer without caffeine (- caffeine); white circles capture a particular cell in the fluorescence micrographs for better clarity of the viewer. The change in relative fluorescence intensity of BP inside the cells with time in absence (b) and presence (c inset) of caffeine where bold lines are the exponential fits of the experimental data points within $15 \%$ error. (c) The fluorescence intensity of BP outside the cells at different time intervals upon the addition of caffeine (+ caffeine) where bold line is the guide to the eye. (d) Caffeine dose-dependent washing of BP from squamous epithelial cells in $5 \mathrm{~min}$ of incubation time. Inset of the figure shows the micrograph of a cell before and after washing with $140 \mathrm{mM}$ caffeine solution. All the experiments are repeated three times.

lining of human mouth) along with their corresponding bright field images taken at 10 and $180 \mathrm{~s}$ following treatment with caffeine (+ caffeine) and with buffer without caffeine (- caffeine) as control. As evident from the fluorescence micrographs, BP stains both the nuclei and the cytoplasms unlike ethidium (Et) and DAPI, which are known to stain specifically the nuclei of the cells (Banerjee et al., 2011). The ability of BP to stain the whole cells can be attributed to its interactions with both DNAs and proteins as evident from one of our recent studies (Banerjee et al., 2013a). Our control experiments suggest that BP emission from the squamous epithelial cells undergoes significant photobleaching with time, and the rate of photo-bleaching has been determined by plotting the relative fluorescence intensity of BP in the cell against time as shown in Figure $5(b)$ and has been found out to be $74 \mathrm{~s}$ from its exponential fit. After addition of caffeine, there is significant BP emission outside the cells as evident from the fluorescence micrographs shown in Figure 5(a). Such BP emission from outside the cells just after the addition of caffeine is a clear signature of caffeine-mediated exclusion of BP from the cells upon forming the caffeine-BP complex. Figure 5(c) shows the time-dependent emission intensity of BP outside the cells following the caffeine treatment. The first micrograph that can be taken next to the caffeine treatment is after $10 \mathrm{~s}$, which includes the time required for adjusting the focus. As evident from Figure 5(c), most of the BP is released from the cells within the first $10 \mathrm{~s}$ at a very fast rate beyond the experimental 
resolution. After $10 \mathrm{~s}, \mathrm{BP}$ is slowly released, which is reflected in the slow enhancement of the emission intensity of BP outside the cells within $10-180 \mathrm{~s}$. Figure 5 (c) inset shows the relative fluorescence intensity inside the cells with respect to that outside the cells against time (within 10-180 s), and the exponential decrease of such relative fluorescence intensity with time further signifies the temporal increase in emission intensity of BP outside the cells because of the release of BP molecules. Such exponential decrease of the relative fluorescence intensity of BP can be fitted with a time constant of $127 \mathrm{~s}$, which signifies the time constant for slow release of BP from the cells after $10 \mathrm{~s}$ of caffeine treatment. As time-dependent relative fluorescence intensity of BP has been monitored inside the cells with respect to that outside the same, the change in emission intensity due to some extent of photo-bleaching in presence of caffeine is nullified. Here, it has to be noted that photo-bleaching of BP in presence of caffeine is significantly reduced as caffeine-BP complexation prevents ET reaction between $\mathrm{BP}$ and biomolecules inside the cells. However, in the analysis shown in Figure 5(c) inset, a significant component has been missed as most of the BP is released from the cells within the first $10 \mathrm{~s}$ after the addition of caffeine, which falls beyond our experimental time resolution. A caffeine dose-dependent extraction of BP from the cell lines in 5 min incubation time is evident from Figure $5(\mathrm{~d})$. An efficient extraction of BP at caffeine concentration of $140 \mathrm{mM}$ is clearly evident from the inset of Figure 5(d).

Upon monitoring the role of caffeine in releasing BP from squamous epithelial cells, such activity of caffeine has also been studied in fibroblast cells (WI-38). Figure 6(a) shows the bright field and fluorescence micrographs of BP-stained WI-38 cells taken at 10 and $70 \mathrm{~s}$ after the treatment with caffeine (+ caffeine) and with buffer without caffeine (- caffeine) as control. Similar to the observation in case of squamous epithelial cells, significant photo-bleaching of BP occurs in the control set of experiments within $10-70 \mathrm{~s}$. The plot of relative fluorescence intensity of BP inside the WI-38 cells versus time in absence of caffeine as shown in Figure 6(b) reveals a time constant of $32 \mathrm{~s}$ of such photo-bleaching from its exponential fit. Addition of caffeine leads to the release of BP resulting in BP emission from exterior of the cells as evident from the fluorescence micrographs (Figure 6(a)) and from the plot of temporal emission intensity of BP outside the cells after the addition of caffeine as shown in Figure 6(c). It is evident from the figure that a significant amount of BP is released from the WI-38 cells within the first $10 \mathrm{~s}$ after the addition of caffeine. Figure $6(\mathrm{c})$ inset shows the plot of relative emission intensity of BP inside the cell with respect to that outside the same versus time following the caffeine treatment. The exponential fit of such plot reveals time constant of $18 \mathrm{~s}$, which signifies the characteristic time of caffeine-mediated BP release from the cells after $10 \mathrm{~s}$ of caffeine treatment.

The caffeine-mediated release of BP has been checked not only in normal cells but also in some cancerous cell lines like breast cancer (MCF-7) and cervical cancer (HeLa) cells, as shown in Figure 7. Both the bright field and fluorescence micrographs of BP-stained MCF-7 and HeLa cells taken at 10 and $120 \mathrm{~s}$ upon the treatment with caffeine (+ caffeine) and with buffer without caffeine (- caffeine) are shown in Figure 7. The fluorescence

(a)

WI38 (fibroblast) cells stained with BP
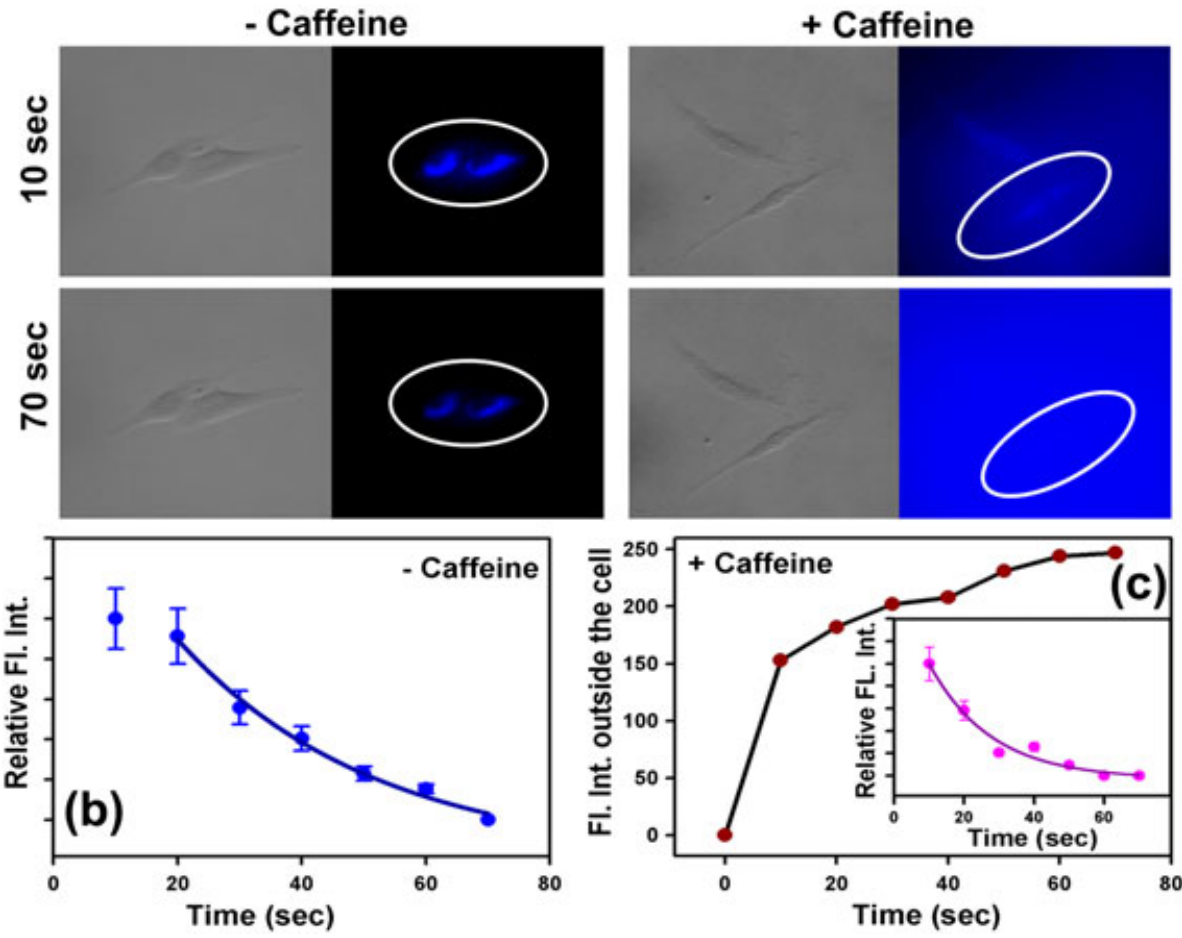

Figure 6. (a) Fluorescence micrographs of BP-stained WI-38 (fibroblast) cells taken at 10 and $70 \mathrm{~s}$ after the addition of caffeine (+ caffeine). Images in grayscale show the respective bright field micrographs. As a control set of experiments, micrographs were taken at similar timescales upon the addition of buffer without caffeine (- caffeine); white circles capture cell(s) in the fluorescence micrographs for better clarity of the viewer. The change in relative fluorescence intensity of $\mathrm{BP}$ inside the cells with time in absence (b) and presence (c inset) of caffeine where bold lines are the exponential fits of the experimental data points within 15\% error. (c) The fluorescence intensity of BP outside the cells at different time intervals upon the addition of caffeine (+ caffeine) where bold line is the guide to the eye. All the experiments are repeated three times. 


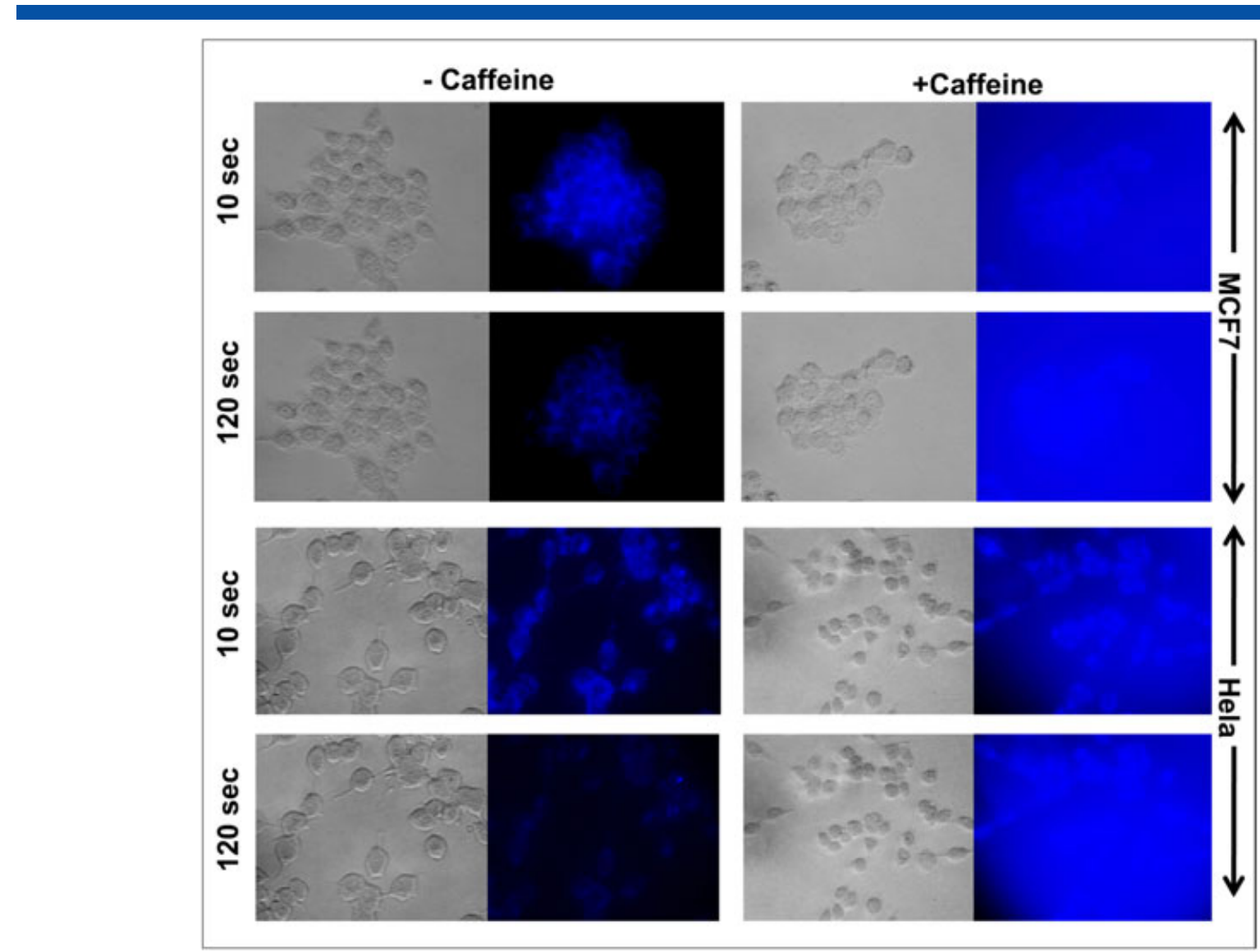

Figure 7. Fluorescence micrographs of BP-stained MCF-7 (breast cancer) and HeLa (cervical cancer) cells taken at 10 and $120 \mathrm{~s}$ upon the addition of caffeine (+ caffeine) and buffer without caffeine (- caffeine). Images in grayscale show the respective bright field micrographs. All the experiments are repeated three times with similar results.

micrographs of control studies (- caffeine) show significant photo-bleaching of BP similar to the observation with normal cells of squamous epithelium and WI-38. Upon treatment with caffeine, $\mathrm{BP}$ is released from both the cancerous cell lines of MCF-7 and HeLa, which results in the enhancement of BP emission from the exterior of the corresponding cells as evident from Figure 7.

\section{CONCLUSION}

Our present study emphasizes the role of caffeine on the biomolecular recognition of benzo[a]pyrene (BP) interrupting the ET pathway from BP to DNA under UVA radiation. The steady-state emission and absorption spectroscopy results suggest that caffeine can host extremely hydrophobic BP molecules in aqueous medium by forming stacked sandwich type structures of caffeine-BP-caffeine complexes as revealed by the molecular modeling studies. The formation of caffeine-BP complexes further leads to the dissociation of
BP from DNA, and thus, the ET from BP to DNA under UVA radiation is inhibited in presence of caffeine as observed from our steady-state and picosecond-resolved emission spectroscopy results. Such caffeine-mediated prevention of ET from BP to DNA consequently protects the DNA from reductive DNA damage as monitored through our AGE. The cellular studies reveal the efficacy of caffeine in dissociating BP from both normal and cancerous cell lines. Our current study may find significance in the potential therapeutic use of caffeine for preventing DNA damage/DNA DSBs and eventually preventing cell death that arises because of such DNA damages.

\section{Acknowledgements}

S. B. thanks UGC for research fellowship. S. C. thanks CSIR, India, for research fellowship. We thank DST for a financial grant (DST/TM/SERI/2k11/103 and SB/S1/PC-011/2013).

\section{REFERENCES}

Banerjee S, Bhowmik D, Verma PK, Mitra RK, Sidhhanta A, Basu G, Pal SK. 2011. Ultrafast spectroscopic study on caffeine mediated dissociation of mutagenic ethidium from synthetic DNA and various cell nuclei. J. Phys. Chem. B 115(49): 14776-14783.

Banerjee S, Verma PK, Mitra RK, Basu G, Pal SK. 2012a. Probing the interior of self-assembled caffeine dimer at various temperatures. J. Fluoresc. 22(2): 753-769.
Banerjee S, Tachiya M, Pal SK. 2012b. Caffeine-mediated detachment of mutagenic ethidium from various nanoscopic micelles: an ultrafast förster resonance energy transfer study. J. Phys. Chem. B 116(27): 7841-7848. Banerjee S, Sarkar S, Lakshman K, Dutta J, Pal SK. 2013a. UVA radiation induced ultrafast electron transfer from a food carcinogen benzo[a] pyrene to organic molecules, biological macromolecules and inorganic nano structures. J. Phys. Chem. B 117(14): 3726-3737. 
Banerjee S, Goswami N, Pal SK. 2013b. A potential carcinogenic pyrene derivative under forster resonance energy transfer to various energy acceptors in nanoscopic environments. ChemPhysChem 14(15): 3581-3593.

Beck SC, Cramb DT. 2000. Condensed phase dispersive interactions of benzo[a]pyrene with various solvents and with DNA: a twist on solvatochromism. J. Phys. Chem. B 104(12): 2767-2774.

Boyland E, Green B. 1962. The interaction of polycyclic hydrocarbons and purines. Br. J. Canc. 16(2): 347-360.

Brown RS, Szolar OHJ, Luong JHT. 1996. Cyclodextrin-aided capillary electrophoretic separation and laser-induced fluorescence detection of polynuclear aromatic hydrocarbons (PAHs). J. Mol. Recognit. 9(5-6): 515-523.

Cadet J, Douki T, Ravanat JL, Mascio PD. 2009. Sensitized formation of oxidatively generated damage to cellular DNA by UVA radiation. Photochem. Photobiol. Sci. 8(7): 903-911.

Carlucci L, Gavezzotti A. 2005. Molecular recognition and crystal energy landscapes: an X-ray and computational study of caffeine and other methylxanthines. Chem. Eur. J. 11(1): 271-279.

Colis LC, Raychaudhury P, Basu AK. 2008. Mutational Specificity of $\gamma$ radiation-induced guanine thymine and thymine guanine intrastrand cross-links in mammalian cells and translesion synthesis past the guanine thymine lesion by human DNA polymerase $\eta$. Biochemistry 47(31): 8070-8079.

Cortez D. 2003. Caffeine inhibits checkpoint responses without inhibiting the ataxia-telangiectasia-mutated (ATM) and ATM- and Rad3-related (ATR) protein kinases. J. Biol. Chem. 278(39): 37139-37145.

Davies DB, Veselkov DA, Djimant LN, Veselkov AN. 2001. Hetero-association of caffeine and aromatic drugs and their competitive binding with a DNA oligomer. Eur. Biophys. J. 30(5): 354-366.

Fredholm BB, Battig K, Holmen J, Nehlig A, Zvartau EE. 1999. Actions of caffeine in the brain with special reference to factors that contribute to its widespread use. Pharmacol. Rev. 51(1): 83-133.

Fujitsuka M, Majima T. 2012. Hole and excess electron transfer dynamics in DNA. Phys. Chem. Chem. Phys. 14(32): 11234-11244.

Geacintov NE, Prusik T, Khosrofian JM. 1976. Properties of benzopyreneDNA complexes investigated by fluorescence and triplet flash photolysis techniques. J. Am. Chem. Soc. 98(21): 6444-6452.

Girard PM, Francesconi S, Pozzebon M, Graindorge D, Rochette P, Drouin R, Sage E. 2011. UVA-induced damage to DNA and proteins: direct versus indirect photochemical processes. J. Phys. Conf. 261: 1-10.

de Gruijl FR. 2000. Photocarcinogenesis: UVA vs UVB. Methods Enzymol. 319: 359-366.

Kuluncsics Z, Perdiz D, Brulay E, Muel' B, Sage E. 1999. Wavelength dependence of ultraviolet-induced DNA damage distribution: involvement of direct or indirect mechanisms and possible artefacts. J. Photochem. Photobiol. B 49(1): 71-80.

Lakowicz JR. 1999. Principles of Fluorescence Spectroscopy. Kluwer Academic/Plenum: New York

Larsen JS, Waluk J, Eriksson S, Thulstrup EW. 1992. Electronic states of benzo[a]pyrene. Linear and magnetic circular dichroism, polarized fluorescence, and quantum chemical calculations. J. Am. Chem. Soc. 114(6): 1942-1949.

Larsen RW, Jasuja R, Hetzler RK, Muraoka PT, Andrada VG, Jameson DM. 1996 Spectroscopic and molecular modelling studies of caffeine complexes with DNA intercalators. Biophys. J. 70(1): 443-452.
Le Pecq JB. 2006. Use of ethidium bromide for separation and determination of nucleic acids of various conformational forms and measurement of their associated enzymes. Methods Biochem. Anal. 20(1): 41-86.

Lu QB. 2010. Effects and applications of ultrashort-lived prehydrated electrons in radiation biology and radiotherapy of cancer. Mutat. Res. 704(1-3): 190-199.

Lu Y, Morimoto K. 2009. Is habitual alcohol drinking associated with reduced electrophoretic DNA migration in peripheral blood leukocytes from ALDH2-deficient male Japanese? Mutagenesis 24(4): 303-308.

Lyles MB, Cameron IL, Rawls HR. 2001. Structural basis for the binding affinity of xanthines with the dna intercalator acridine orange. J. Med. Chem. 44(26): 4650-4660.

Marcus RA. 1993. Electron transfer reactions in chemistry: theory and experiment (Nobel lecture). Angew. Chem. Int. Ed. Engl. 32: 1111-1121.

Marta RA, Wu R, Eldridge KR, Martens JK, McMahon TB. 2010. Infrared vibrational spectra as a structural probe of gaseous ions formed by caffeine and theophylline. Phys. Chem. Chem. Phys. 12(14): 3431-3442.

Nguyen J, Ma Y, Luo T, Bristow RG, Jaffray DA, Lu QB. 2011. Direct observation of ultrafast-electron-transfer reactions unravels high effectiveness of reductive DNA damage. Proc. Natl. Acad. Sci. 108(29): 11778-11783.

Nielsen PE. 1990. Chemical and photochemical probing of DNA complexes. J. Mol. Recognit. 3(1): 1-25.

Nosaka Y, Akasaka K, Hatano H. 1978. Interaction of benzo[a]pyrene and 6-oxybenzo[a]pyrene with caffeine. Structures of the complexes as studied by nuclear magnetic resonance chemical shift and relaxation. Structures of the complexes as studied by nuclear magnetic resonance chemical shift and relaxation. J. Phys. Chem. 82(26): 2829-2833.

Pal SK, Zhao L, Zewail AH. 2003. Water at DNA surfaces: ultrafast dynamics in minor groove recognition. Proc. Natl. Acad. Sci. 100(14): 8113-8118.

Phillips DH. 1983. Fifty years of benzo(a)pyrene. Nature 303(5917): 468-472.

Rafael J, Carmona L, Galano A. 2011. Is caffeine a good scavenger of oxygenated free radicals? J. Phys. Chem. B 115(15): 4538-4546.

Sage E, Girard PM, Francesconi S. 2012. Unravelling UVA-induced mutagenesis. Photochem. Photobiol. Sci. 11: 74-80.

Sarkar R, Pal SK. 2006. Ligand-DNA interaction in a nanocage of reverse micelle. Biopolymers 83(6): 675-686.

Toyooka T, Ibuki Y, Koike M, Ohashi N, Takahashi S, Goto R. 2004. Coexposure to benzo[a]pyrene plus UVA induced DNA double strand breaks: visualization of Ku assembly in the nucleus having DNA lesions. Biochem. Biophys. Res. Commun. 322(2): 631-636.

Toyooka T, Ibuki Y, Takabayashi F, Goto R. 2006. Coexposure to benzo[a] pyrene and UVA induces DNA damage: first proof of double-strand breaks in a cell-free system. Environ. Mol. Mutagen. 47(1): 38-47.

Traganos F, Kaminska-Eddy B, Darzynkiewicz Z. 1991. Caffeine reverses the cytotoxic and cell kinetic effects of Novantrone (mitoxantrone). Cell Prolif. 24(3): 305-319.

Villringer A, Chance B. 1997. Non-invasive optical spectroscopy and imaging of human brain function. Trends Neurosci. 20(10): 435-442.

Wang CR, Nguyen J, Lu QB. 2009. Bond breaks of nucleotides by dissociative electron transfer of nonequilibrium prehydrated electrons: a new molecular mechanism for reductive DNA damage. J. Am. Chem. Soc. 131(32): 11320-11322. 